\title{
Hepatocellular Carcinoma in a Tertiary Referral Hospital in Indonesia: Lack of Improvement of One-Year Survival Rates between 1998-1999 and 2013-2014
}

\author{
Imelda M Loho*, Irsan Hasan, C Rinaldi A Lesmana, Esthika Dewiasty, Rino \\ A Gani
}

\begin{abstract}
Background: The survival of hepatocellular carcinoma (HCC) patients is usually low due to late diagnosis. Cipto Mangunkusumo Hospital as the largest tertiary referral hospital in Indonesia, has recently improved its modalities for advanced HCC management, but there has not been any evaluation on any improvement in HCC patient survival. Materials and Methods: A retrospective analysis on 114 HCC patients in 2013-2014 were conducted and compared with the database for 77 HCC patients in 1998-1999. Clinical characteristics and treatment received were recorded and the survival of both groups was analyzed using the Kaplan-Meier method and compared using the log-rank test. Results: The percentage of $\mathrm{HBV}$ positive patients had increased after fifteen years from $32.5 \%$ to $67.5 \%$. Only two patients $(1.8 \%)$ in $2013-2014$ were diagnosed with HCC during surveillance program. Proportions of Barcelona Clinic Liver Cancer A, B, C, and D in 2013-2014 were $1.8 \%, 42 \%, 28.1 \%$, and $28.1 \%$, respectively. There was an increase in the use of potentially curative treatment, such as surgical resection or combination of loco-regional therapies. The one-year survival rate increased from $\mathbf{2 4 . 1 \%}$ in $1998-1999$ to $29.4 \%$ in $2013-2014$, while the median survival decreased from 146 days to 138 days, but the difference was not statistically significant $(p=0.913)$. Conclusions: There was no improvement in the median survival of HCC patients after fifteen years because most continued to present at late stages. There is an urgent need for a nationwide implementation of a hepatitis screening program and HCC surveillance education.
\end{abstract}

Keywords: Hepatocellular carcinoma - survival - 1998-1999 - 2013-2104 - Indonesia

Asian Pac J Cancer Prev, 17 (4), 2165-2170

\section{Introduction}

Hepatocellular carcinoma (HCC) is the second most common cause of death from cancer worldwide and a major health problem in South-Eastern Asia.(Ferlay et al.) Data regarding HCC patients survival in Southeast Asian countries, such as Thailand and Malaysia, has shown that the survival of HCC patients is still very low due to delay in diagnosis.(Sithinamsuwan et al., 2000; Norsa'adah and Nurhazalini-Zayani, 2013; Somboon et al., 2014) $\mathrm{HCC}$ is also a major health problem in Indonesia, as the biggest country in Southeast Asia. Fifteen years ago, HCC patients survival in Cipto Mangunkusumo Hospital, as the largest referral hospital for HCC patients management in Indonesia, was also very low with median survival of 4.8 months and one-year survival rate $24.1 \%$. (Mulyana, 2001) Recently, more advanced HCC treatment options have been available in this hospital. Therefore, we wanted to evaluate whether there has been any improvement in the survival of HCC patients who came in 2013-2014 compared to those who came in 1998-1999.

\section{Materials and Methods}

A retrospective database analysis from $\mathrm{HCC}$ patients diagnosed between 2013-2014 in Cipto Mangunkusumo Hospital was conducted and compared to the database from HCC patients who were diagnosed in 1998-1999. (Mulyana, 2001) Information on patients' characteristics, type and frequency of treatment, were collected. If there was no data regarding patients' death in the medical records, the patients were contacted by phone and if they could not be reached, the patients were censored on the day of their last visit. Diagnosis of cirrhosis was made by clinical evaluations (presence of liver cirrhosis stigmata, such as spider angioma, ascites, palmar erythema, asterixis, etc), ultrasound (US) evaluations, endoscopic findings suggesting the presence of portal hypertension, and laboratory features (AST to Platelet Ratio (APRI) score $>1,2$ ).(Lin et al., 2008)

Patients were also classified whether they were diagnosed based on recent findings under surveillance program or whether they were diagnosed due to their

Internal Medicine Department, Faculty of Medicine, Universitas Indonesia, Jakarta, Indonesia *For correspondence: imelda. loho@gmail.com 
symptoms. Diagnosis was confirmed by combining a diagnostic value of AFP ( $\geq 200 \mathrm{ng} / \mathrm{mL}$ ) with typical features in one imaging technique (dynamic computed tomography (CT) scan or magnetic resonance imaging (MRI) or contranst enhanced US (CEUS).(Omata et al., 2010) In the absence of diagnostic AFP elevation, diagnosis was confirmed by typical features in two imaging techniques or by histopathology examination.(Omata et al., 2010) Cancer was staged according to Barcelona Clinic Liver Cancer (BCLC) staging system(Llovet et al., 1999; 2012a) in 2013-2014 and according to the American Joint Committee on Cancer 5th edition of the American Joint Committee on Cancer (AJCC) TNM system(1997) in 1998-1999. The BCLC stage for patients in 1998-1999 could not be calculated because secondary data was used and there would be a high risk of inaccuracy in defining the performance status retrospectively. Investigations to detect metastases were performed when extra-hepatic involvement was suspected. Cancer gross pathology was classified as nodular if the margin of the tumor was clearly defined and diffuse if the margin was not clearly defined. Tumor size was classified according to the largest diameter of a single nodule $(<3 \mathrm{~cm}, 3-5 \mathrm{~cm}$, and $>5 \mathrm{~cm})$.

\section{Treatment classification}

Patients were classified into three main groups according to the treatment protocol: curative, palliative, or supportive group. Curative group consisted of liver transplantation, surgical resection, percutaneous ethanol injection therapy (PEIT), and radiofrequency ablation (RFA). Palliative group consisted of transarterial chemoembolization (TACE), transarterial chemo-infusion (TACI), sorafenib, immunochemotherapy, and tamoxifen, while supportive group included patients who did not receive any specific treatment for their HCC. If patients received combination of treatment in which one of the modalities was potentially curative (liver transplantation, surgical resection, RFA, or PEIT), they will be classified into curative group, otherwise they will be categorized in the palliative group.

\section{Statistical analysis}

Continous data are expressed as mean value \pm standard deviation (SD) if the distribution is normal and as median value with minimal and maximal value if the distribution is not normal. Categorical variables are expressed as absolute and relative frequencies. The actuarial survivals were calculated according to the Kaplan-Meier method and compared by the log-rank test. The one-year survival rate is reported. A two-tailed $p$ value $<0.05$ was considered statistically significant. Statistical analysis was performed using the SPSS 20.0 statistical package.

\section{Ethics}

This study was approved by the Ethics Committee of the Faculty of Medicine, Universitas Indonesia.

\section{Results}

From 182 patients who had HCC diagnosis according to our hospital database in 2013-2014, there were 114 patients were excluded because of various reasons (Figure 1). In period 1998-1999, there was no data on number of patients excluded and the reasons for exclusion (Figure 2 ). Occurrence of HCC is still dominated by males in both periods, while the median of age decreased from 60 years in 1998-1999 to 54 years in 2013-2014 (Table 1). The number of $\mathrm{HBV}$ patients increased after fifteen years and HBV became the most common etiology of HCC in 20132014, while the number of non-B non-C HCC decreased. There was an increase in the frequency of Child-Pugh A but there was also an increase in Child-Pugh $\mathrm{C}$ patients in 2013-2014.

Most patients in 2013-2014 came to seek medical help after symptoms had occurred. In $93.9 \%$ patients, clinical symptoms had already occurred when they were diagnosed with HCC. The most common clinical signs or symptoms were hepatomegaly $(76.3 \%)$, weight loss $(68.4 \%)$, abdominal pain $(64.9 \%)$, jaundice $(43.9 \%)$, ascites $(36.8 \%)$, and hematemesis or melena (17\%). From 114 patients in 2013-2014, 109 patients were referred to our hospital after they were diagnosed with HCC and only five patients had routinely visited our outpatient clinic before they were diagnosed with HCC. Of those who were referred to our hospital, none of them had been diagnosed with HCC during surveillance process and only seven of

\section{Table 1. Patient Characteristics}

\begin{tabular}{|c|c|c|}
\hline Variables & $1998-1999$ & 2013-2014 \\
\hline Number of patients: $\mathrm{n}(\%)$ & $77(40.3)$ & $114(59.7)$ \\
\hline \multicolumn{3}{|l|}{ Sex: $\mathrm{n}(\%)$} \\
\hline Male & $62(80.5)$ & $95(83.3)$ \\
\hline Female & $15(19.5)$ & $19(16.7)$ \\
\hline Age (years), (range) & $60(29-82)$ & $54(18-83)$ \\
\hline \multicolumn{3}{|l|}{ Age Group: n (\%) } \\
\hline$<60$ years & $37(48.1)$ & $83(72.8)$ \\
\hline$\geq 60$ years & $40(51.9)$ & $31(27.2)$ \\
\hline \multicolumn{3}{|c|}{ Referral from other hospital: $\mathrm{n}(\%)$} \\
\hline Yes & No data & $109(95.6)$ \\
\hline No & No data & $5(4.4)$ \\
\hline \multicolumn{3}{|c|}{ Presence of clinical symptoms at diagnosis: $\mathrm{n}(\%)$} \\
\hline Yes & No data & $107(93.9)$ \\
\hline No & No data & $7(6.1)$ \\
\hline \multicolumn{3}{|c|}{ Diagnosis under surveillance program: $\mathrm{n}(\%)$} \\
\hline Yes & $\mathrm{NA}^{*}$ & $2(1.8)$ \\
\hline No & $\mathrm{NA}^{*}$ & $112(98.2)$ \\
\hline \multicolumn{3}{|l|}{ Etiology: n (\%) } \\
\hline Hepatitis B & $25(32.5)$ & $77(67.5)$ \\
\hline Hepatitis C & $22(28.6)$ & $16(14)$ \\
\hline Non-B non-C & $26(33.8)$ & $20(17.5)$ \\
\hline \multicolumn{3}{|c|}{ Hepatitis B and Hepatitis C coinfection } \\
\hline & $4(5.2)$ & $0(0)$ \\
\hline No data & $0(0)$ & $1(1)$ \\
\hline \multicolumn{3}{|c|}{ Presence of liver cirrhosis: $\mathrm{n}(\%)$} \\
\hline Yes & $72(93.5)$ & $89(78.1)$ \\
\hline No & $4(5.2)$ & $15(13.2)$ \\
\hline No data & $1(1.3)$ & $10(8.7)$ \\
\hline \multicolumn{3}{|c|}{ Child-Pugh classification: $\mathrm{n}(\%)$} \\
\hline A & $16(20.8)$ & $53(46.5)$ \\
\hline B & $39(50.6)$ & $31(27.2)$ \\
\hline $\mathrm{C}$ & $6(7.8)$ & $30(26.3)$ \\
\hline No data & $16(20.8)$ & $0(0)$ \\
\hline
\end{tabular}

NA: not applicable, $\mathrm{P}$ : regular surveillance with ultrasound and alpha feto-protein had not been applied and no data was collected 
HCC in a Tertiary Referral Hospital in Indonesia: One-Year Survival Rates in 1998-1999 and 2013-2014

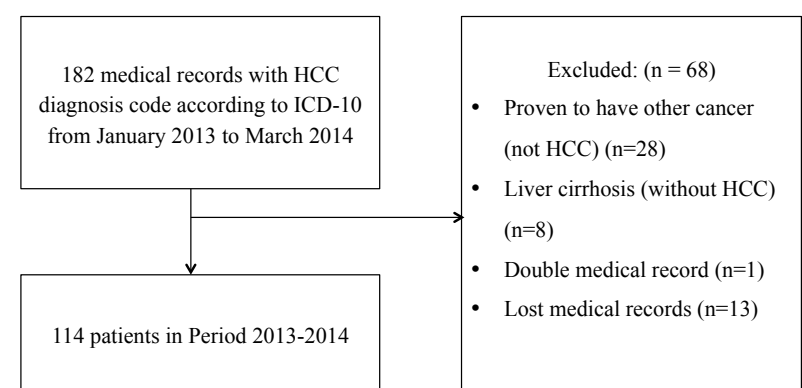

Figure 1. Flow Diagram Showing the Patient Selection in Period 2013-2014

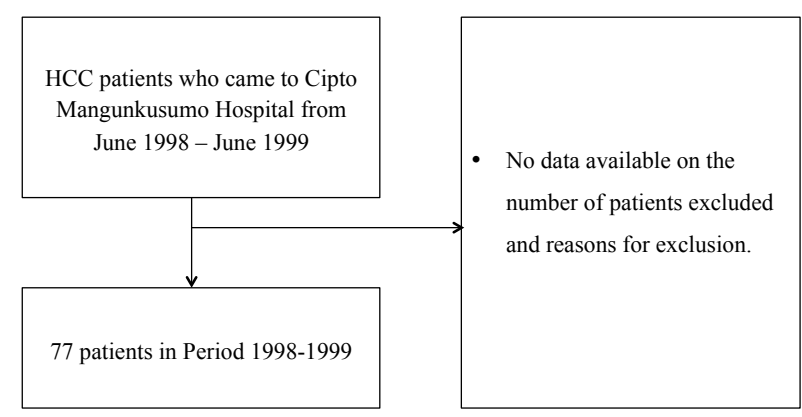

Figure 2. Flow Diagram Showing the Patient Selection in Period 1998-1999

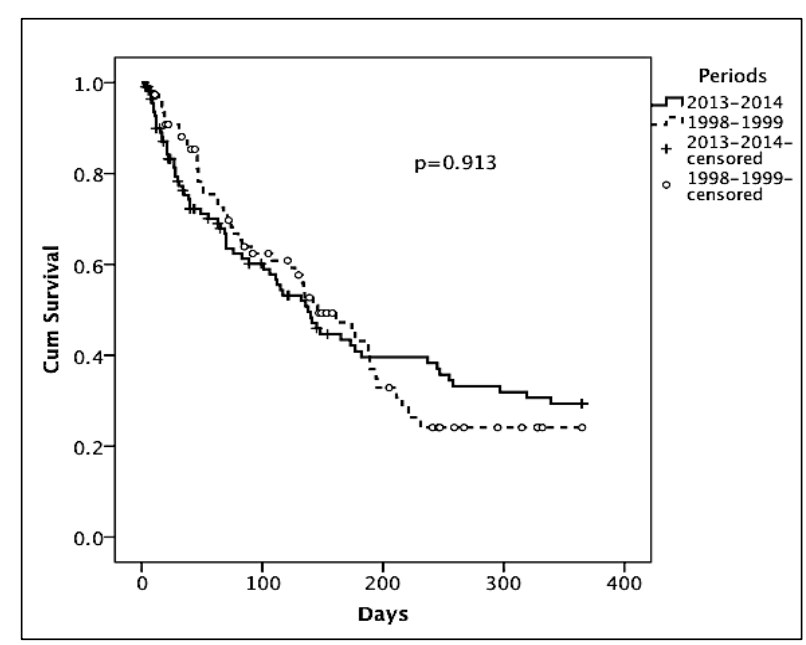

Figure 3. Cumulative survival of HCC patients which showed lack of median survival improvement between 1998-1999 and 2013-2014

them had already known their hepatitis status. From five patients who had routinely visited our hospital, only two patients were diagnosed during surveillance process. One patient was diagnosed in BCLC stage B with tumor size of $7.5 \mathrm{~cm}$ and the other was diagnosed in BCLC stage $C$ with tumor size of $6.1 \mathrm{~cm}$ but with lymph node enlargement.

In both periods, most patients came in late stages (Table 2). In 1998-1999,93.5\% of patients were diagnosed in TNM stage III or IV according to the fifth edition of American Joint Committee on Cancer (AJCC), while in 2013-2014, 28.1\% were diagnosed in BCLC stage C and $28.1 \%$ were diagnosed in BCLC stage D. About $65-$ $67 \%$ of patients in both groups could only get supportive treatment (Table 3). Surgical therapies were done in seven patients in 2013-2014 while no patients got surgical
Table 2. Features of Hepatocellular Carcinoma

\begin{tabular}{|c|c|c|}
\hline Variables & $1998-1999$ & $2013-2014$ \\
\hline $\begin{array}{l}\text { Largest tumor diameter: } \\
\text { mean }(\mathrm{SD})(\mathrm{cm})\end{array}$ & $6.2( \pm 3.4)$ & $10.9( \pm 4.9)$ \\
\hline \multicolumn{3}{|c|}{ Classification of largest tumor diameter: $\mathrm{n}(\%)$} \\
\hline$<3 \mathrm{~cm}$ & $7(9.1)$ & $2(1.8)$ \\
\hline $3-5 \mathrm{~cm}$ & $2(2.6)$ & $3(2.6)$ \\
\hline$>5 \mathrm{~cm}$ & $50(64.9)$ & $108(94.7)$ \\
\hline No data & $18(23.4)$ & $1(0.9)$ \\
\hline \multicolumn{3}{|l|}{ Type of Tumor } \\
\hline Nodular & $61(79.2)$ & $84(73.7)$ \\
\hline Diffuse & $16(20.8)$ & $27(23.7)$ \\
\hline No data & $0(0)$ & $3(2.6)$ \\
\hline \multicolumn{3}{|c|}{ Number of Tumor Nodules: n, (\%) } \\
\hline Single & & $62(54.4)$ \\
\hline Multiple & $\mathrm{NA} \pm$ & $49(43)$ \\
\hline No data & & $3(2.6)$ \\
\hline \multicolumn{3}{|c|}{ Presence of portal vein thrombus: $\mathrm{n}(\%)$} \\
\hline Yes & $20(26)$ & $34(29.8)$ \\
\hline No & $57(74)$ & $67(58.8)$ \\
\hline No data & $0(0)$ & $13(11.4)$ \\
\hline \multicolumn{3}{|l|}{ AFP level: n (\%) } \\
\hline$<200 \mathrm{ng} / \mathrm{mL}$ & $39(50.6)$ & $35(30.7)$ \\
\hline$\geq 200 \mathrm{ng} / \mathrm{mL}$ & $37(48.1)$ & $70(61.4)$ \\
\hline No data & $1(1.3)$ & $9(7.9)$ \\
\hline \multicolumn{3}{|l|}{ BCLC staging: n (\%) } \\
\hline 0 & & $0(0)$ \\
\hline A & & $2(1.8)$ \\
\hline B & $\mathrm{NA}^{\#}$ & $48(42)$ \\
\hline $\mathrm{C}$ & & $32(28.1)$ \\
\hline $\mathrm{D}$ & & $32(28.1)$ \\
\hline \multicolumn{3}{|c|}{ TNM staging according to AJCC fifth edition: $\mathrm{n}(\%)$} \\
\hline Stage I & $0(0)$ & \\
\hline Stage II & $5(6.5)$ & \\
\hline Stage III (IIIA and IIIB) & $49(63.6)$ & NA* \\
\hline Stage IVA & $17(22.1)$ & \\
\hline Stage IVB & $6(7.8)$ & \\
\hline
\end{tabular}

AFP: alpha feto-protein, AJCC: American Joint Committee on Cancer, BCLC: Barcelona Clinic Liver Cancer, HCC: hepatocellular carcinoma, NA, not applicable, \pm : in 1998-1999, data on number of nodules was not recorded separately, but was included in the TNM stage, ${ }^{*}$ : Patients could not be classified according to BCLC stage because performance status was not recorded, *: TNM stage was not applied in 2013-2014.

treatment in 1998-1999. In three of the seven patients, transarterial chemoembolization (TACE) and PEIT were done before surgical procedures. The use of TACE as single treatment increased from six patients $(7.8 \%)$ to 13 patients (11.4\%). Combination of two modalities or more was the most common type of treatment given in 20132014 (Table 3). Combination therapies consisted of two or more of the followings, i.e. surgical resection, RFA, PEIT, TACE, TACI, sorafenib, and radiotherapy. These patients were classified in one group because there were many types of combination with only very small number of patients in each type.

Seventeen BCLC stage B patients could not undergo TACE procedure because of the long waiting list. Some of them died while they were waiting for their TACE schedule and some of them were lost-to-follow up. In this study, the waiting time varied between 10-190 days. In patients with portal vein thrombus or BCLC C patients, six patients were given transarterial chemoinfusion (TACI) and one patient was given combination of sorafenib and TACI. The use of sorafenib was very limited because the 
Table 3. Treatment of Hepatocellular Carcinoma

\begin{tabular}{lcc}
\hline \multicolumn{1}{c}{ Treatment modalities } & $1998-1999$ & $2013-2014$ \\
\hline Treatment modalities: $\mathrm{n}(\%)$ & 0 & 0 \\
Liver transplantation & 0 & $4(3.5)$ \\
Resection & $3(3.9)$ & 0 \\
PEIT & 0 & 0 \\
RFA & $6(7.8)$ & $13(11.4)$ \\
TACE & $1(1.3)$ & $6(5.3)$ \\
TACI & 0 & 0 \\
Sorafenib & $8(10.4)$ & 0 \\
Tamoxifen & $2(2.6)$ & 0 \\
Immuno-chemotherapy & & \\
Combination of two & 0 & $16(14)$ \\
modalities or more: resection, & & \\
RFA, PEIT, TACE, TACI, & & 0 \\
sorafenib, radiotherapy & $5(6.5)$ & $75(65.8)$ \\
Combination of TACE/TACI & $52(67.5)$ & \\
with immuno-chemotherapy & Supportive treatment &
\end{tabular}

PEIT: percutaneous ethanol injection, RFA: radiofrequency ablation, TACE: transarterial chemoembolization, TACI: transarterial chemoinfusion, Immuno-chemotherapy: interferon and 5-fluorourasil

cost was very high and it has not been covered by the national health insurance.

\section{Survival}

The one-year survival rate increased from $24.1 \%$ in 1998-1999 to $29.4 \%$ in $2013-2014$, while the median survival decreased from 146 days in 1998-1999 to 138 days in 2013-2014 (Fig. 3), but the difference was not statistically significant $(p=0,913)$. Stratified analyses for different type of treatment and for surveillance and nonsurveillance group were not conducted because there was only small number of patients in each group.

\section{Discussion}

To our knowledge, this is the first study from a Southeast Asian country, which compared survival of HCC patients in two different periods. This study showed several changes in clinical features of HCC after fifteen years. Based on western studies, (Santi et al., 2012),(Hucke et al., 2011) HCC is more common encountered in older patients, while in our study, the median age of HCC patients decreased from 60 to 54 years. This might be explained by the different pattern of etiology. Hepatitis B virus (HBV) is the most common etiology of HCC in our study, as in other studies from Southeast Asia,(Sithinamsuwan et al., 2000; Lucas et al., 2009; Norsa'adah and Nurhazalini-Zayani, 2013; Somboon et al., 2014) while hepatitis C virus (HCV) is the most common etiology in western countries.(ElSerag et al., 2003; Santi et al., 2012) Chronic hepatitis $\mathrm{B}$ patients might suffer from $\mathrm{HCC}$ at younger age than chronic hepatitis C.(Hiotis et al., 2012) Interestingly, the pattern of decreasing age is not found in two different studies from Thailand. In 2000, the mean age at diagnosis was 54, (Sithinamsuwan et al., 2000) while in 2014, the mean age was 57.(Somboon et al., 2014) This might be explained by the decreasing percentage of chronic hepatitis $\mathrm{B}$ and the increasing percentage of chronic hepatitis C.(Sithinamsuwan et al., 2000; Somboon et al., 2014) Although this two studies took place in different regions in Thailand, the decreasing number of hepatitis $\mathrm{B}$ is in contrary with our result. Our result showed an increasing number of hepatitis B from $32.5 \%$ to $67.5 \%$. This was probably due to an increase in hepatitis B prevalence in Indonesia from $8.8 \%$ in 1995 (Sulaiman et al., 1995) to $9.4 \%$ in 2012(2012b) and better coverage of national health insurance program which improves the opportunities for every patients from rural areas to be referred to a tertiary hospital.

After fifteen years, there had been improvement in the number of patients who underwent TACE, surgical resection, and combination of RFA with other modalities. Despite not statistically significant, there was an improvement in one-year survival rate from $24.1 \%$ in $1998-1999$ to $29.4 \%$ in $2013-2014$. In contrary, the median survival declined from 146 days in 1998-1999 to 138 days in 2013-2014. One-year survival rate is the percentage of people in a study or treatment group who are still alive for one year after they were diagnosed with or started treatment for a disease. Median survival is the length of time from either the date of diagnosis or the start of treatment for a disease, such as cancer, that half of the patients in a group of patients diagnosed with the disease are still alive. In this study, one-year survival rate improved because the percentage of patients treated with potentially curative treatment, such as surgical resection, was higher in 2013-2014 than in 1998-1999. In contrary, the median survival declined because the percentage of patients diagnosed in advanced stage in 2013-2014 was higher than in 1998-1999. Therefore the length of time from date of diagnosis that half of the patients were still alive is shorter.

In 2013-2014, although the majority of patients were diagnosed in BCLC B and only $1.8 \%$ were diagnosed in BCLC A, the total number of patients with BCLC C and BCLC D was higher and they were not appropriate candidates for TACE, RFA, and surgical resection. This pattern is slightly different from Thailand where most patients are diagnosed in BCLC B, but the percentage of BCLC A is $16 \%$ and BCLC 0 is $9.6 \%$. (Somboon et al., 2014) One important factor that contributed to this result was the low number of HCC patients diagnosed under surveillance process in our study. In a study from Taiwan,(Kuo et al., 2010) the overall survival was better for patients in surveillance group compared with non-surveillance group because larger proportions of patients in the surveillance group possessed small tumors and afforded more curative treatment options. To our knowledge, there has been no data from Southeast Asian countries regarding the proportion of HCC patients who were diagnosed during surveillance process.

In Japan, where nationwide surveillance has been applied in high-risk population since $1980,62 \%$ of HCC cases were diagnosed in BCLC A.(Kudo, 2012) Surveillance was not only conducted by tertiary referral centers, but also by small hospitals and private practitioners. This could happen because Japanese government actively involved in preventive measures against hepatitis and HCC. Free testing for hepatitis B 
and $\mathrm{C}$ virus was offered in public health centers and medical facilities nationwide. To improve awareness of the importance of early HCC detection, the Japanese Society of Hepatology also designated a person in each prefecture for providing educational lectures. As a result, Japan has the best median survival time for HCC patients among other countries in the world, such as US and Spain.(Kudo, 2012; Johnson et al., 2015) The latest data on median survival of HCC patients in Japan is 47.2 months, while the median survival of HCC patients in the US was only 18.6 months and in Spain was only 26 months.(Johnson et al., 2015)

Several studies in US and Europe have demonstrated that surveillance for HCC in high-risk patients is being underutilized in clinical practice.(Davila et al., 2010; Rosa et al., 2010; Davila et al., 2011; Singal et al., 2011; Singal et al., 2012) Surveillance rate was only $18,4 \%$ and was significantly higher among patients followed in subspecialty gastroenterology clinics.(Singal et al., 2012) The difference between Japan and other countries was the government involvement in the education and preventive measures. Without continuous education and government policy on HCC surveillance, screening of high-risk patients will only be done in institutional level only.

There were three factors that might contribute to the low level of HCC surveillance in our study, i.e. lack of physicians' awareness on the importance of HCC surveillance in high-risk population, lack of national policy on HCC surveillance, and lack of chronic hepatitis $\mathrm{B}$ and hepatitis $\mathrm{C}$ detection in our population. Adequate knowledge on how and when-to-do HCC surveillance, should be owned by all primary care physicians who would encounter more high-risk patients in their daily practice than physicians in tertiary care. In this study, some patients with known HCC risk factors never entered surveillance system before they were diagnosed with HCC. To overcome this, continuous education on HCC surveillance should be conducted in all provinces in Indonesia. Physicians' awareness on HCC surveillance should also be accompanied by a clear national policy from the ministry of health to regulate the follow-up and referral system if an HCC nodule was found. To improve detection of high-risk population, screening of hepatitis $\mathrm{B}$ and hepatitis $\mathrm{C}$ should also be a part of national policy.

Our study has several limitations. First, as this is a retrospective study, there is naturally some missing data on several patients' characteristics. Second, classification of patients according to BCLC stage could not be made in the 1998-1999-group because BCLC stage had not been applied during that time. Recoding would result in improper staging because no data regarding performance status was collected during that period. Third, the survival of patients in the surveillance group and nonsurveillance group could not be compared using log-rank test because there were only a small number of patients in the surveillance group. On the other hand, our result is very important for clinicians and decision makers in the healthcare field to increase their awareness of the importance of HCC surveillance and screening of hepatitis $\mathrm{B}$ and hepatitis $\mathrm{C}$.

In conclusion, despite the availability of advanced treatment modalities, there was no improvement in the median survival of HCC patients because most patients came in late stages. Therefore, implementation of nationwide hepatitis screening program for general population and HCC surveillance education for primary physicians and high-risk population, should be part of national health policies.

\section{Acknowledgements}

No grant supports have been received. The authors acknowledge Edy Mulyana, MD, for his contribution in providing 1998-1999 database spreadsheet.

\section{References}

NCI Dictionary of Cancer Terms [Online]. National Cancer Institute.

1997. AJCC Cancer Staging Manual, Philadelphia, LippincottRaven. (2012a). EASL-EORTC clinical practice guidelines: management of hepatocellular carcinoma. J Hepatol, 56, 908-43.

2012b. [Guidelines on Viral Hepatitis Control], Jakarta, Directorate General of Disease Control and Health Restructuring Ministry of Health, Republic of Indonesia [Article in Indonesian].

Davila JA, Henderson L, Kramer JR, et al (2011). Utilization of surveillance for hepatocellular carcinoma among hepatitis $\mathrm{C}$ virus-infected veterans in the United States. Ann Intern Med, 154, 85-93.

Davila JA, Morgan RO, Richardson PA, et al (2010). Use of surveillance for hepatocellular carcinoma among patients with cirrhosis in the United States. Hepatol, 52, 132-41.

El-Serag HB, Davila JA, Petersen NJ, et al (2003). The continuing increase in the incidence of hepatocellular carcinoma in the United States: an update. Ann Intern Med, 139, 817-23.

Hiotis SP, Rahbari NN, Villanueva GA, et al (2012). Hepatitis $\mathrm{B}$ vs. hepatitis $\mathrm{C}$ infection on viral hepatitis-associated hepatocellular carcinoma. BMC Gastroenterol, 12, 64.

Hucke F, Sieghart W, Schoniger-Hekele M, et al (2011). Clinical characteristics of patients with hepatocellular carcinoma in Austria - is there a need for a structured screening program? Wien Klin Wochenschr, 123, 542-51.

Johnson PJ, Berhane S, Kagebayashi C, et al (2015). Assessment of liver function in patients with hepatocellular carcinoma: a new evidence-based approach-the ALBI grade. J Clin Oncol, 33, 550-8.

Kudo M (2012). Japan's Successful Model of Nationwide Hepatocellular Carcinoma Surveillance Highlighting the Urgent Need for Global Surveillance. Liver Cancer, 1, 141-3.

Kuo YH, Lu SN, Chen CL, et al (2010). Hepatocellular carcinoma surveillance and appropriate treatment options improve survival for patients with liver cirrhosis. Eur $J$ Cancer, 46, 744-51.

Lin CS, Chang CS, Yang SS, et al (2008). Retrospective evaluation of serum markers APRI and AST/ALT for assessing liver fibrosis and cirrhosis in chronic hepatitis B and $\mathrm{C}$ patients with hepatocellular carcinoma. Intern Med, 47, 569-75.

Llovet JM, Bru C, Bruix J (1999). Prognosis of hepatocellular carcinoma: the BCLC staging classification. Semin Liver Dis, 19, 329-38.

Lucas ZDF, Pangan CP, Patal PC, et al (2009). The clinical profile of hepatocellular carcinoma patients at the Philippine General Hospital. Phil J Internal Med, 47, 1-9. 
Norsa'adah B, Nurhazalini-Zayani CG (2013). Epidemiology and survival of hepatocellular carcinoma in north-east Peninsular Malaysia. Asian Pac J Cancer Prev, 14, 6955-9.

Omata M, Lesmana L, Tateishi R, et al (2010). Asian Pacific Association for the Study of the Liver consensus recommendations on hepatocellular carcinoma. Hepatol International, 4, 439-74.

Rosa I, Denis J, Renard P, et al (2010). A French multicentric longitudinal descriptive study of hepatocellular carcinoma management (The Changh Cohort): preliminary results. $J$ Hepatol, 52, S231-2.

Santi V, Buccione D, Di Micoli A, et al (2012). The changing scenario of hepatocellular carcinoma over the last two decades in Italy. $J$ Hepatol, 56, 397-405.

Singal AG, Volk ML, Rakoski MO, et al (2011). Patient involvement in healthcare is associated with higher rates of surveillance for hepatocellular carcinoma. J Clin Gastroenterol, 45, 727-32.

Singal AG, Yopp A, C SS, et al (2012). Utilization of hepatocellular carcinoma surveillance among American patients: a systematic review. J Gen Intern Med, 27, 861-7.

Sithinamsuwan P, Piratvisuth T, Tanomkiat W, et al (2000). Review of 336 patients with hepatocellular carcinoma at Songklanagarind Hospital. World J Gastroenterol, 6, 339-43.

Somboon K, Siramolpiwat S, Vilaichone RK (2014). Epidemiology and survival of hepatocellular carcinoma in the central region of Thailand. Asian Pac J Cancer Prev, 15, 3567-70.

Sulaiman HA, Julitasari, Sie A, et al (1995). Prevalence of hepatitis B and C viruses in healthy Indonesian blood donors. Trans R Soc Trop Med Hyg, 89, 167-70. 